\title{
Doppelbrechungsänderungen bei der aktiven Kontraktion des Fasermodells (aus Kaninchen-Psoas)
}

\author{
Von Gerda Ströbel \\ Aus dem Physiologischen Institut der Universität Tübingen \\ (Z. Naturforschg. 7 b, 102-108 [1952]; eingegangen am 6. Oktober 1951)
}

\begin{abstract}
1. Die Kontraktion Glycerin-Wasser-extrahierter Modellfasern aus dem m. psoas des Kaninchens findet nur bei sehr dünnen Fasern im ganzen Querschnitt gleichmäßig statt. Bei dicken Fasern findet sich bei maximaler Verkürzung ein positiv doppelbrechender Mantel um einen Kern, der in der üblichen Additionslage der Messung negativ doppelbrechend, isotrop oder sehr schwach positiv doppelbrechend erscheint.

2. Die Analyse der Auslöschrichtungen im Mantel und Kern ergibt, daß diese Besonderheiten der Doppelbrechung im Kern auf einer Schlängelung der Ketten der Eiweiß-Stäbchen beruhen. Im Mantel und im ganzen Querschnitt dünner Fasern sind die optische Achse und damit die Ketten der Eiweiß-Stäbchen auch nach der Verkürzung der Faserachse parallel. Bei dicken Fasern wird also offenbar der Faserkern durch die Verkürzung des Fasermantels gestaucht.

3. Bei dickeren Fasern verkürzt sich nur der Fasermantel, weil das ATP nur in den Fasermantel eindringt. Denn die Dicke des aktiven Fasermantels stimmt mit der Grenzschichtdicke der Eindiffusion von ATP überein. Der Kern wird gestaucht, weil er an der Kontraktion nicht teilnimmt.

4. Die Gesamtdoppelbrechung von dünnen Fasermodellen, die keinen Stauchungskern enthalten, ist der Doppelbrechung intakter Muskelfasern gleich - sowohl im unkontrahierten Zustand wie auch nach maximaler Verkürzung.

5. Im unkontrahierten Zustand ist bei Muskel- und Modellfaser auch die Zusammensetzung der Gesamtdoppelbrechung aus Eigen- und Stäbchendoppelbrechung gleich. Es ist anzunehmen, daß das gleiche auch für den verkürzten Zustand gilt.

6. Bei der Verkürzung nimmt die Gesamtdoppelbrechung ab, weil die Eigendoppelbrechung abnimmt. Die Stäbchendoppelbrechung bleibt in allen Imbibitionsmitteln unverändert, außer in Alkohol. Die Änderung in Alkohol beruht offenbar auf einer Nebenreaktion.

7. Also beruht die Verkürzung der Modell- und Muskelfaser auf einer Verkürzung der einzelnen Eiweiß-Stäbchen, deren Lage dabei parallel der Faserachse bleibt. Auch die verkürzten Eiweiß-Stäbchen sind immer noch so lang, daß sie den Bedingungen des Wienerschen Stäbchenmischkörpers genügen.

8. In Stauchungszonen, in denen die Richtung der Eiweiß-Stäbchen der Faserachse nicht mehr parallel ist, vermindern sich dagegen Eigen- und Stäbchendoppelbrechung proportional - bis zu negativen Werten.

9. Die Verkürzung der Faser ist mit einer Verdickung verbunden. Aber die Querschnittszunahme ist kleiner als die Längenabnahme. Bei maximaler Verkürzung (auf 20-30\% der Anfangslänge) beträgt das Endvolumen etwa $60 \%$ des Anfangsvolumens.
\end{abstract}

I.

$\mathrm{B}_{\mathrm{c}}^{\mathrm{c}}$ ei der Muskelkontraktion nimmt die Doppelbrechung ab, wenig bei isometrischer Kontraktion ${ }^{1}$, sehr beträchtlich dagegen, wenn Verkürzung erlaubt ist $^{2}$. Das Ausmaß der Doppelbrechung ist also bei der Muskelkontraktion ganz überwiegend bestimmt durch den Grad der Längenänderung und nicht oder nur unwesentlich durch den Übergang aus dem inaktiven in den aktiven Zustand. Es erhebt sich die

1 A. v. M u r a l t, Pfiügers Arch. ges. Physiol. 230, 299 [1932].

2 v. E b n e r, Pflügers Arch. ges. Physiol. 163, 179 [1916].
Frage, auf welcher Änderung der Feinstruktur die Abhängigkeit der Doppelbrechung von der Muskellänge beruht.

Es ist polarisationsoptisch ${ }^{3}$ und röntgenoptisch ${ }^{4}$ gesichert, daß die passiven Längenänderungen von Aktomyosinsystemen nicht auf Richtungsänderungen, sondern ausschließlich auf Längenänderungen der einzelnen Eiweiß-Stäbchen beruhen. Es ist die Frage, ob das gleiche für die aktiven Längenänderungen

3 H. H. W e be r, Pflügers Arch. ges. Physiol. 235. 205-233 [1934].

4 W. T. A s tbury u. S. Dickenson, Proc. Roy. Soc. [London], Ser. B 129, 307 [1940]. 
gilt. Falls diese Frage polarisationsoptisch beantwortet werden soll, muß ermittelt werden, wie sich die Änderung der Doppelbrechung bei aktiver Kontraktion auf Eigen- und Stäbchendoppelbrechung verteilt. Das ist möglich bei der ATP-Kontraktion des sogenannten Fasermodells von A. W e b e ${ }^{5}$.

\section{II.}

Die Wasser-Glycerin-extrahierte Einzelfaser des m. psoas des Kaninchens wird nach Befestigung in einem Dilatometer ${ }^{5}$ durch ATP zur Kontraktion gebracht und kann in jedem beliebigen Verkürzungszustand nach Entfernung des ATP fixiert werden (durch 5-proz. Formaldehyd).. Die Doppelbrechung solcher verkürzter Fasern wird dann in verschiedenen Flüssigkeiten, deren Brechungsindices zwischen 1,33 und 1,69 variieren, gemessen und mit der Doppelbrechung fixierter, aber unkontrahierter und unverkürzter Fasern verglichen.

In der unkontrahierten Modellfaser ist die Doppelbrechung positiv. Dies war zu erwarten, denn es ist bei der lebenden Muskelfaser ${ }^{6}$ einerseits und beim voll geordneten Faden aus hochgereinigtem Aktomyosin ${ }^{3}$. andererseits ebenso. Für die kontrahierte Modellfaser gilt das gleiche zuverlässig nur für die äußeren Schichten bis zu einer Tiefe von 10-15 $\mu$ von der Oberfläche der Faser aus. Wir nennen diese äußeren Schichten den Fasermantel. Ist der Radius der Modellfaser wesentlich größer als $10-15 \mu$, so ist im Kern die Doppelbrechung häufig negativ, fehlt ganz oder ist zumindest als positive Doppelbrechung sehr stark vermindert.

Diese Abweichung der Kerndoppelbrechung dickerer Fasern $(2 \mathrm{r}>30-40 \mu)$ von der normalen positiven Doppelbrechung beruht nicht auf einer Verwandlung der Eiweiß-Stäbchen in Eiweiß-Kugeln oder Eiweiß-Plättchen.

Wird nämlich die kontrahierte Modellfaser mit ihrer Längsachse der Schwingungsrichtung des Analysators oder Polarisators parallel gestellt, so wird die Doppelbrechung des Fasermantels gelöscht, die Doppelbrechung des Kerns dagegen nicht. Im Fasermantel der kontrahierten Modellfaser liegt also ebenso wie in der lebenden Muskelfaser oder im geordneten Aktomyosinfaden die optische Achse parallel zur geometrischen Achse. Im Faserkern der kontrahierten Modellfaser ist dies offenbar nicht der Fall.

Wird die Modellfaser in der angegebenen Lage durch ein Rot-I-Plättchen betrachtet, so zerfällt der

5 A. W e b e r, Biochimica et Biophysica Acta 7, 214 [1951].

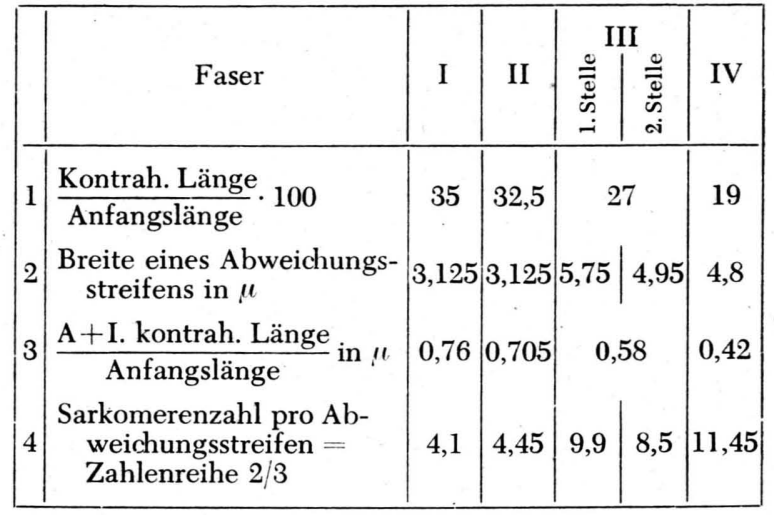

Tab. 1. Vergleich der mittleren Höhe der kontrahierten Sarkomere mit der Höhe eines Abweichungsstreifens.

leuchtende Kern in eine Folge von Querstreifen (Abweichungsstreifen), die alternierend Additionsfarbe und Subtraktionsfarbe tragen. Dies spricht dafür, daß die optische Achse von Streifen zu Streifen alternierend nach rechts und links von der geometrischen Achse abweicht.

In Muskelfasern und anderen geordneten Aktomyosinsystemen repräsentiert die optische Achse die Richtung der untereinander parallelen EiweißStäbchen. Also scheinen die Ketten der Eiweiß-Stäbchen im Kern einer kontrahierten Modellfaser nicht mehr gestreckt, sondern geschlängelt zu verlaufen. Diese Deutung wird erhärtet, wenn man die Auslöschrichtung der einzelnen Streifen aufsucht, in die der Faserkern in der angegebenen Lage zerfällt. Es bestätigt sich, daß diese Auslöschrichtung (die Richtung der optischen Achse) abwechselnd nach rechts und links von der einheitlichen Auslöschrichtung des Mantels abweicht. Die Schlängelung ist in ihrem Ausmaß verschieden: stärker und schwächer gefaltete oder geschlängelte Bezirke folgen längs der Faserachse aufeinander.

\section{III.}

Die einzelnen Sarkomere sind zwar in der unkontrahierten Modellfaser, aber nicht mehr in der kontrahierten Modellfaser zu erkennen. Dies mag z. Tl. darauf beruhen, daß die A- und I-Banden durch die Kontraktion sehr schmal geworden $\operatorname{sind}(\mathrm{A}+\mathrm{I}<0,7 \mu)$, z. Tl. auf der gestörten Ordnung der Struktur, durch die die A- und I-Banden der einzelnen Fibrillen nicht mehr genau in der gleichen Höhe liegen. Infolgedessen wird die Höhe der kontrahierten Sarkomere aus dem Wert vor der Kontraktion berechnet.

6 D. Noll u. H. H. Web e r, Pflügers Arch. ges. Physiol. 235, 234-246 [1934]. 


\begin{tabular}{|c|c|c|c|c|c|}
\hline & \multicolumn{2}{|c|}{ Drehung um die Faserachse } & $0^{\circ}$ & $90^{\circ}$ & $180^{\circ}$ \\
\hline \multirow{2}{*}{ I } & \multirow{2}{*}{$\begin{array}{l}\text { Farbe der gemessenen Stelle mit Rot-I-Plätt- } \\
\text { chen, wenn sich der Fasermantel in } \\
\text { a) Additionslage befindet } \\
\text { b) Fasermantel in Auslöschrichtung durch } \\
\text { Drehung aus der Additionslage um } 45^{\circ} \\
\text { im Sinne des Uhrzeigers }\end{array}$} & \multirow[t]{2}{*}{$\begin{array}{l}\text { Linke Hälfte des gemess. Streifens } \\
\text { Rechte Hälfte des gemess. Streifens }\end{array}$} & $\begin{array}{l}\text { rot- } \\
\text { (violett) } \\
\text { orange }\end{array}$ & $\begin{array}{l}\text { gelb } \\
\text { gelb }\end{array}$ & $\begin{array}{l}\text { orange } \\
\text { rot- } \\
\text { (violett) }\end{array}$ \\
\hline & & & gelb & rot & blau \\
\hline \multirow{2}{*}{ II } & \multirow{2}{*}{$\begin{array}{l}\text { Daraus Richtung der Abweichung der Eiweiß- } \\
\text { Stäbchen von der Faserachse (positiv im } \\
\text { Sinne des Uhrzeigers, negativ entgegen) }\end{array}$} & Linke Hälfte des gemess. Steifens & positiv & $\sim 90^{\circ}$ & negativ \\
\hline & & Rechte Hälfte des gemess. Streifens & positiv & $\sim 90^{\circ}$ & negativ \\
\hline \multirow{2}{*}{ III } & \multirow{2}{*}{$\begin{array}{l}\text { Winkel zwischen Auslöschrichtung und Faser- } \\
\text { achse, d. h. Winkel zwischen Eiweiß-Stäb- } \\
\text { chen und Faserachse }\end{array}$} & Linke Hälfte des gemess. Streifens & $+31^{\circ}$ & $\sim 90^{\circ}$ & $-59^{\circ}$ \\
\hline & & Rechte Hälfte des gemess. Streifens & $+63^{\circ}$ & $\sim 90^{\circ}$ & $-32^{\circ}$ \\
\hline
\end{tabular}

Tab. 2. Auslöschrichtungen (Richtungen der Eiweiß-Stäbchen) an derselben Stelle des Faserkernes bei Drehung der Faser um die Längsachse (und zwar um $90^{\circ}$ und $180^{\circ}$ ).

Es ergibt sich, daß etwa 5-10 Sarkomere in einer Schicht einsinniger Achsenabweichung enthalten sind (Tab. 1, Reihe 4). Die bis hierher nachgewiesene Faltung oder Schlängelung erscheint also verhältnismäßig grob und regelmäßig.

Die Schlängelung erweist sich als sehr viel unregelmäßiger und komplizierter, wenn ein einzelner Streifen mit hoher Vergrößerung (nominell 800-fach, effektiv 640-fach) genau auf seine Auslöschrichtung analysiert wird. Dann erweist sich die Auslöschrichtung in verschiedenen Teilen - etwa in der rechten und linken Hälfte des Streifens - als deutlich verschieden (Abb. 1; Tab. 2, I und III). Außerdem nimmt die Abweichung der Teilchenrichtung von der Faserachse im allgemeinen an der Grenze zum Fasermantel deutlich ab. Besonders stark und unregelmäßig ändern sich Auslöschrichtung und Achsen der Eiweiß-Stäbchen in demselben Streifen, wenn die Modellfaser um ihre Längsachse gedreht wird, so daß man die Stäbchenbündel einmal gewissermaßen von oben, das andere Mal von der Seite und schließlich von unten sieht (Abb. 1). Die Verschiedenheiten sind so groß, daß es schwierig oder unmöglich wird, den Verlauf der Ketten von Eiweiß-Stäbchen räumlich dreidimensional richtig zu konstruieren.

Dreht man die Faser dagegen genau um $180^{\circ}$ um ihre Längsachse und sorgt durch Markierung mit Kohlestäubchen dafür, daß vorher und nachher exakt dieselbe Stelle beobachtet wird, so findet man in Anfangs- und Endstellung eine ungefähr spiegelbildliche Symmetrie der Auslöschrichtungen, d. h. der Achsenrichtungen der Eiweiß-Teilchen (Tabelle 2, Spalte $0^{\circ}$ und $180^{\circ}$ ).

Dieses ganze Bild, besonders aber die spiegelbildliche Symmetrie der Stäbchenrichtungen sichert es, daß die Abnahme der positiven Doppelbrechung und der Umschlag in negative Doppelbrechung im Kern der kontrahierten Faser nicht auf einer Formänderung der Eiweiß-Stäbchen, sondern auf einer Richtungsänderung durch Faltung und Schlängelung der Ketten beruht. Wenn die Richtung der Eiweiß-Stäbchen von der geometrischen Faserachse gerade um $45^{\circ} \mathrm{ab}$ weicht, so wird die Kerndoppelbrechung in der nor-

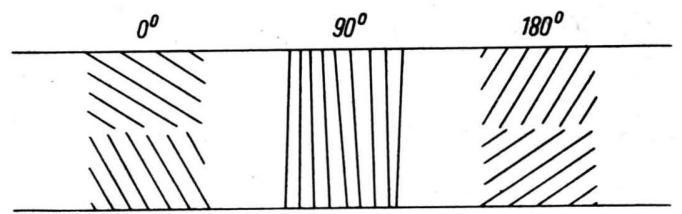

Abb. 1. Verlaufsrichtung der Eiweiß-Stäbchen in ein und demselben Abweichungsstreifen eines gestauchten Faserkernes bei Drehung um die Längsachse der Faser. Die obere und untere Begrenzung gibt die Richtung der Faserachse, die Schraffierung die Richtung der Eiweil3Stäbchen. Die drei nebeneinander dargestellten Abweichungsstreifen sind in Wirklichkeit Bilder desselben Streifens, und zwar unter $0^{\circ}$ in der Ausgangsstellung, unter $90^{\circ}$ nach Drehung um einen rechten Winkel, unter $180^{\circ}$ nach Drehung um zwei rechte Winkel. Die gemessenen Abweichungswinkel der Stäbchenachse vor der Faserachse finden sich in Tab. 2.

malen Meßlage - d. h. unter $45^{\circ}$ in Additionslage Null; wenn die Richtung der Stäbchen mehr als $45^{\circ}$ abweicht, wird die Kerndoppelbrechung in Additionslage negativ.

Die achsenparallele Anordnung der Eiweiß-Stäbchen bei der Kontraktion dünner Fasern und bei der Kontraktion des Mantels dicker Fasern steht im Gegensatz zu der Schlängelung der Eiweiß-Ketten im Kern dicker Fasern. Dieser Gegensatz legt den Gedanken nahe, es handle sich hier um eine Stauchung des passiven Kerns durch den aktiv sich kon- 


\begin{tabular}{|c|c|c|c|c|}
\hline Doppelbre & Modell & Muskel & Modell & Muskel \\
\hline & \multicolumn{2}{|c|}{ kontrahiert } & \multicolumn{2}{|c|}{ unkontrahiert } \\
\hline & & & & \\
\hline & & - & & \\
\hline amtdoppelbr. & 1,44 & $1,4^{11}$ & 2,7 & $2,6^{10}$ \\
\hline
\end{tabular}

Tab. 3. Vergleich der Doppelbrechung von intakten Muskelfasern und Modellfasern in gestrecktem und kontrahiertem Zustand.

trahierenden Mantel. Diese Ansicht gewinnt an Sicherheit, wenn berechnet wird, wie tief ATP bei der gegebenen Spaltungsrate in die Faser eindringen kann. Wird die auf anderem Weg bestimmte Diffusionskonstante des ATP im Gewebe der Modellfaser mit $30-40 \cdot 10^{-9}$ und die experimentell bestimmte Spaltungsrate in die Formel von Me yer h of eingesetzt, so berechnet sich tatsächlich eine Eindringungstiefe $\sim 15 \mu-$ d. h. die Dicke der Mantelschicht ${ }^{7}$.

\section{IV.}

Infolgedessen kann nur die Doppelbrechung des Fasermantels oder - praktischer und einfacher die Doppelbrechung von Modellfasern ohne Stauchungskern zur Beurteilung der Strukturänderung bei aktiver Kontraktion herangezogen werden. Dann zeigt sich (Tab. 3), daß die Gesamtdoppelbrechung der Modellfaser in kontrahiertem und unkontrahiertem Zustand der Gesamtdoppelbrechung der kontrahierten und unkontrahierten lebenden Muskelfaser praktisch gleich ist. Das gleiche gilt für die Stäbchenund Eigendoppelbrechung der unkontrahierten Modellfaser und der unkontrahierten intakten Muskelfaser (Tab. 3). Ebenso ist der Brechungsindex der Eiweiß-Stäbchen $\left(n_{1}\right)$ für die intakte Muskelfaser $\left(n_{1}=1,57^{6}\right)$, die Modellfaser $\left(n_{1}=1,56\right)$ und auch den Aktomyosinfaden $\left(n_{1}=1,57^{3}\right)$ praktisch gleich. Die Feinstruktur der Modellfaser scheint der Feinstruktur der intakten Muskelfaser also in allen Kontraktionszuständen gleich zu sein. Danach ist es berechtigt anzunehmen, daß auch die Verteilung der Doppelbrechung auf Eigen- und Stäbchendoppelbrechung für die kontrahierte Muskelfaser nicht anders ist als für die kontrahierte Modellfaser.

Kurve 1 auf der linken Seite der Abb. 2 zeigt, daß die Stäbchendoppelbrechung unkontrahierter Fasern von Faser zu Faser nur wenig streut. Größer ist da-

7 W. H a s selb a ch, Deutsche Physiologentagung, August 1951, Mainz; noch nicht veröffentlicht.

10 E. F i s c h e r, Ann. New York Acad. Sci. 47, 783 [1947].

11 vo n E b n e r, Pflügers Arch. ges. Physiol. 163, 179 [1916]. gegen die Streuung der Gesamtdoppelbrechung, weil die Eigendoppelbrechung (d. h. das Minimum der Gesamtdoppelbrechung) einigermaßen verschieden ist. Das Minimum der niedrigsten Doppelbrechungskurve (Kurve 2) liegt etwa 40\% tiefer als das Minimum der

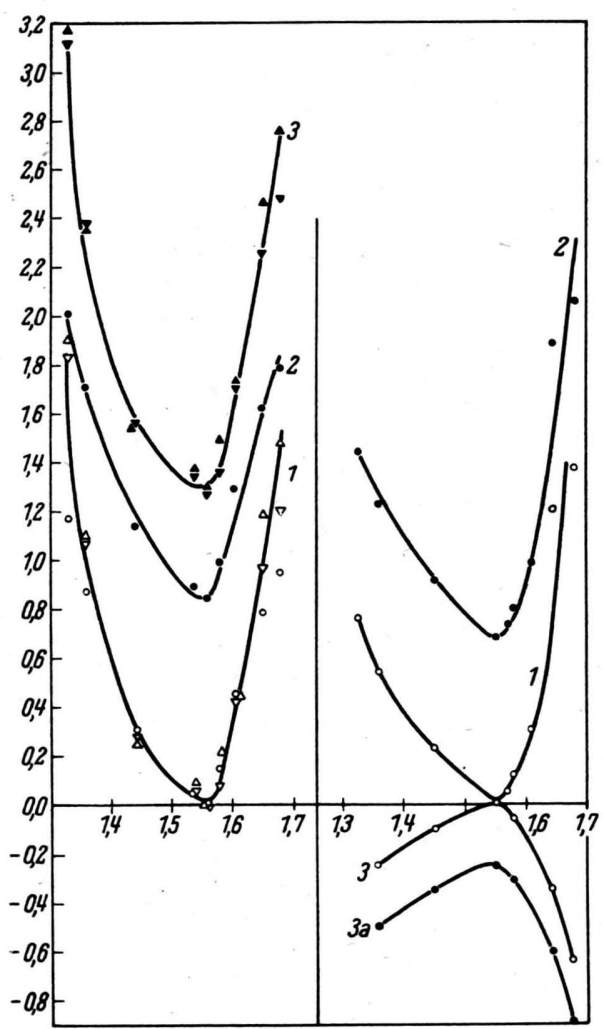

Abb. 2. 'Gesamtdoppelbrechung und Stäbchendoppelbrechung bei unkontrahierten Modellfasern: linke Seite, und kontrahierten Modellfasern: rechte Seite. Ordinate : Doppelbrechung $\times 10^{3} ; \mathrm{A}$ b s z i s s e : Brechungsindices der Imbibitionsflüssigkeiten. L in k e $\mathrm{S}$ e it e : Kurve 2 und 3 Maximal- und Minimalwerte der Gesamtdoppelbrechung. von 6 Fasern $(\boldsymbol{\Delta} \nabla \bullet$ Versuchspunkte). Kurve 1 Stäbchendoppelbrechung der Fasern von Kurve 2 und 3 ( $\Delta \nabla \circ$ Versuchspunkte). R e ch te S e it e : Kurve 2 Durchschnittswert der Gesamtdoppelbrechung von $4 \mathrm{Fa}$ sern nach Kontraktion um 66-77\%. Kurve 1 Anteil der Stäbchendoppelbrechung. Kurve 3 a negative Gesamtdoppelbrechung an einer, "gestauchten“ Stelle (s. Text). Kurve 3 die dazugehörige Stäbchendoppelbrechung.

höchsten Kurve (Kurve 3; die dazwischen liegenden Doppelbrechungskurven 3 weiterer Fasern sind der Übersichtlichkeit wegen weggelassen). Diese Streuung dürfte wahrscheinlich darauf beruhen, daß es nicht immer gelingt, schwache Kontraktionen der Modellfaser beim Einbringen der lebenden Muskelfasern in die Extraktionsmischung aus Wasser und Glycerin zu vermeiden ${ }^{5}$. 


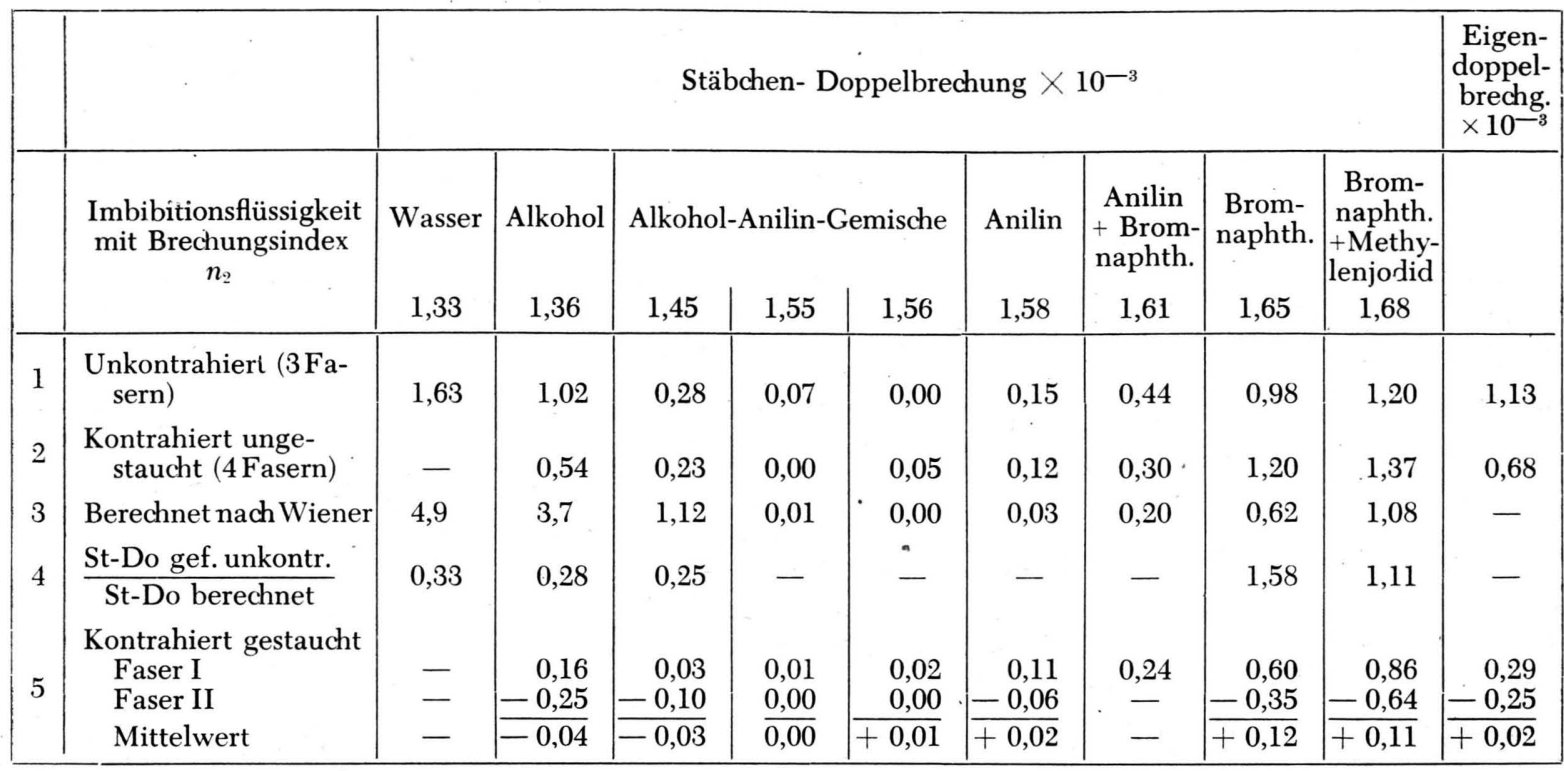

Tab. 4. Eigen- und Stäbchendoppelbrechung unkontrahierter und kontrahierter Modellfasern auf einem mittleren Eiweißgehalt von $15 \%$ umgerechnet*.

Denn die Mittelwertskurve der Doppelbrechung von vier ungestauchten kontrahierten Fasern (Abb. 2, rechte Seite, Kurve 2) zeigt, daß bei der Kontraktion vorzugsweise die Eigendoppelbrechung abnimmt. Sie sinkt bei einer Kontraktion um $66 \%$ bis $77 \%$ auf annähernd die Hälfte des unkontrahierten Betrages (Tab.4, Reihe 2). Der Mittelwert der Stäbchendoppelbrechung dagegen ist bei unkontrahierter und kontrahierter Faser in allen Imbibitionsmitteln etwa gleich - außer in Alkohol (Tab. 4). Nun ist schon seit langem bekannt ${ }^{3,6}$, daß gerade in Alkohol die Stäbchendoppelbrechung hinter dem theoretischen Wert weit zurückbleibt. Dies beruht darauf, daß Alkohol nicht nur zwischen die Stäbchen, sondern auch in die Stäbchen eindringt. Es liegt infolgedessen nahe, die Abnahme der Stäbchendoppelbrechung in Alkohol als Folge einer Auflockerung der Stäbchen bei der Kontraktion und einer dadurch vergrößerten Alkoholaufnahme anzusehen. Die andere Möglichkeit - Absinken der. Stäbchendoppelbrechung durch Verkürzung der Stäbchen - müßte sich in allen Imbibitionsflüssigkeiten bemerkbar machen.

Die Eigendoppelbrechung sinkt nicht nur scheinbar ab - etwa weil auch in anscheinend ungestauchten Fasern eine submikroskopische Stauchung stattfindet. Denn wenn die Stäbchenachse von der Faserachse auch nur auf submikroskopische Strecken abweicht, muß die Eigen- und Stäbchendoppelbrechung proportional abnehmen und gegebenenfalls sogar in propor- tionalem Betrage negativ werden, falls die einzelnen Abweichungswinkel größer als $45^{\circ}$ sind (vgl. Abschnitt III). Das ist an erkennbar gestauchten Stellen tatsächlich der Fall (Abb. 2, rechte Seite, Kurve 3 und 3 a sowie Tab. 4, Reihe 5). Bei den ungestauchten Fasern aber fällt nur die Eigendoppelbrechung ab, während die Stäbchendoppelbrechung unverändert bleibt.

Es läßt sich also einigermaßen sicher sagen, daß molekular-morphologisch die aktive Kontraktion und die passive Dehnung gleiche Vorgänge entgegengesetzter Richtung sind: Die im einzelnen EiweißStäbchen achsenparallel angeordneten Peptidketten gehen bei der Kontraktion aus einem mittleren in einen stark gefalteten Zustand über, während sie bei der Dehnung gestreckt werden. Das ist die Ursache der Änderung der Eigendoppelbrechung. Die Stäbchendoppelbrechung ändert sich nicht, weil die Stäbchen als Ganzes achsenparallel orientiert bleiben und weil ihre Länge auch in verkürztem Zustand immer noch so groß ist, daß sie den Voraussetzungen der Wienerschen Gleichung für Stäbchenmischkörper praktisch genügt.

Eine gewisse Stütze findet dieses Ergebnis in den elektronen-mikroskopischen Aufnahmen ${ }^{8}$. Die fein-

8 M. H. D raper u. A. J. H o d g e, Austral. J. exp. Biol. med. Sci. 27, 465 [1949], 'sowie C. E. H a ll, M. A. Jakus u. F. O. S chmitt, Biologic. Bull. 90, 32 [1946].

${ }^{*}$ Vgl. Methodik, Berechnungen. 


\begin{tabular}{|c|c|c|c|c|c|c|}
\hline & & $\begin{array}{c}1 \\
\text { Länge } \\
\text { in mm }\end{array}$ & $\begin{array}{c}2 \\
r \operatorname{in} \mu\end{array}$ & $\begin{array}{c}\quad 3 \\
\text { Volumen } \\
\text { in } \mathrm{mm}^{3}\end{array}$ & $\begin{array}{c}4 \\
\text { Länge }\end{array}$ & $\begin{array}{c}5 \\
\text { Anfangswertes } \\
\text { Volumen }\end{array}$ \\
\hline Faser I & $\begin{array}{l}\text { unkontrahiert } \\
\text { kontrahiert }\end{array}$ & $\begin{array}{r}19 \\
4\end{array}$ & $\begin{array}{l}33 \\
57,5\end{array}$ & $\begin{array}{l}0,065 \\
0,041\end{array}$ & 79 & 36 \\
\hline Faser II & $\begin{array}{l}\text { unkontrahiert } \\
\text { kontrahiert }\end{array}$ & $\begin{array}{r}23 \\
7\end{array}$ & $\begin{array}{l}40 \\
54\end{array}$ & $\begin{array}{l}0,116 \\
0,064\end{array}$ & 69,5 & 44,5 \\
\hline Faser III & $\begin{array}{l}\text { unkontrahiert } \\
\text { kontrahiert }\end{array}$ & $\begin{array}{l}32 \\
10\end{array}$ & $\begin{array}{l}30 \\
46\end{array}$ & $\begin{array}{l}0,090 \\
0,066\end{array}$ & 68,8 . & 26 \\
\hline Faser IV & $\begin{array}{l}\text { unkontrahiert } \\
\text { kontrahiert }\end{array}$ & $\begin{array}{r}17 \\
4\end{array}$ & $\begin{array}{l}24 \\
33\end{array}$ & $\begin{array}{l}0,030 \\
0,015\end{array}$ & 73,5 & 50 \\
\hline
\end{tabular}

Tab. 5. Längen- und Querschnittsänderungen der Modellfaser bei der Kontraktion (7 Versuche).

sten elektronen-mikroskopisch noch auflösbaren Eiweißfilamente verlaufen in gedehntem, normalem und kontrahiertem Zustand immer gestreckt und der Faserachse parallel und werden nur als Ganzes länger oder kürzer

Wie beim voll geordneten Aktomyosinfaden ${ }^{3}$ ist die Stäbchendoppelbrechung der Modellfaser in allen Imbibitionsflüssigkeiten mit einem Brechungsindex $n_{2}>1,56$ der nach $\mathrm{W}$ i e $\mathrm{n}$ e $\mathrm{r}$ berechneten theoretischen Stäbchendoppelbrechung praktisch.gleich (Tab. 4, Reihe 4). Bei der intakten Faser des m. cutaneus des Frosches beträgt die gefundene Stäbchendoppelbrechung für $n_{2}>1,56$ nur etwa die Hälfte der berechneten Stäbchendoppelbrechung ${ }^{6}$. Das Verhalten der intakten Muskelfaser ist verständlicher als das der Modellfaser. Denn die Fasern enthalten im Gegensatz zum Aktomyosinfaden außer Aktomyosin auch noch andere Proteinstrukturen, die nicht doppelbrechend sind. Wenn auch der Betrag dieser anderen Eiweißkörper in der Modellfaser durch die Wasser-GlycerinExtraktion vermindert ist, so sollte doch der verbliebene Rest an isotropen Proteinen die Doppelbrechung unter den theoretischen Betrag eines Wienerschen Mischkörpers senken.

\section{A n hang}

Bei der Kontraktion des lebenden Muskels muß das Volumen der Muskelfaser - abgesehen von einer minimalen Volumenstriktion - konstant bleiben, weil die Muskelfaser von einer intakten Membran umgeben ist. Bei der Herstellung der Modellfaser wird diese Membran weitgehend zerstört. Es zeigt sich, daß infolgedessen das Volumen nicht konstant bleibt: Die Querschnittszunahme ist bei hohen Graden der Verkürzung zwar beträchtlich, aber doch geringer als die Längenabnahme (Tab. 5, Spalte 1 u. 2). Infolgedessen beträgt das Volumen nach der Kontraktion nur mehr gut die Hälfte des Volumens vorher (Tab. 5, Spalte 4).

Dieses Verhalten ist verständlich, wenn Muskelfaser und Modellfaser größere Mengen an chemisch nicht gebundenem Wasser enthalten. Die kontraktile Struktur der Modellfaser im Bad kann in diesem Fall vorgestellt werden wie ein System aus zwei Drahtnetzen, die durch eine große Zahl von parallelen Gummifäden miteinander verbunden sind. Das Volumen dieses Systems ist dann begrenzt durch die beiden Drahtnetze und die äußerste Schicht der Gummifäden. Wenn sich die Gummifäden eines solchen Systems in einem Wasserbad zusammenziehen, so wird das Volumen mit der Annäherung der beiden Drahtnetze aneinander kleiner, weil das Wasser zwischen den Gummifäden durch die Drahtnetze austreten kann. Umgibt man das System dagegen mit einer enganliegenden Gummihaut, so bleibt das Volumen bei der Kontraktion der Gummifäden konstant wie das Volumen der intakten Muskelfaser.

\section{Methodik}

Die Präparation der Modellfas ern erfolgte nach A. W e ber ${ }^{5}$.

Die Vorbereitung der Modellfasern für die polarisationsoptischen Untersuchung e n : Für die quantitative Analyse der Doppelbrechung werden die Modellfasern nach $\mathrm{H}$. H. W e ber 3 sowie Noll und We ber ${ }^{6}$ auf Glashaken befestigt. Das Aufkleben selbst wurde in einigen Versuchsreihen modifiziert. Die unkontrahierten Fasern werden nach Imbibition mit Glycerin aufgeklebt. Infolgedessen trocknen sie auch beim Aufkleben in Luft nicht aus. Es war deshalb möglich, als Klebstoff hochprozentige Gelatine anzuwenden, die von den organischen Lösungsmitteln nicht angegriffen wird, und diese Gelatinetropfen nach Eintrocknung mit einer Cellonlackschicht zu überziehen, die die Auflösung der Gelatine in Wasser verhindert. Nach Trocknung der Befestigungsstellen wird die Doppelbrechung in verdünnter Pufferlösung (s. unter Lösungen) gemessen. Dann wird die Messung in 5-10-proz. Formaldehydlösung wiederholt. Die Größe der Doppelbrechung ändert sich durch die Formaldehydeinwirkung nicht.

Die kontrahierten Fasern werden in kontrahiertem $\mathrm{Zu}$ stand sofort durch Formaldehyd fixiert. Sie werden an- 
schließend unter der Oberfläche der Formaldehydlösung mit einem Tropfen hochprozentiger Gelatine auf den Glashäkchen befestigt. Dann wird der Glashaken mit dem Präparat in Alkohol gebracht, um die Gelatine zu härten; die erste Messung der Doppelbrechung findet also erst in Alkohol statt.

Die Untersuchung der Auslöschwinkel und der Staıchungserscheinungen fand in einer flachen Metallwanne mit Glasboden statt, deren Wand an genau gegenüberliegenden Stellen durch zwei um ihre Achse drehbare Metallstifte durchbohrt ist. Ihre Spitzen stehen sich genau zentriert gegenüber. Die Drehung der beiden Metallstifte ist gekoppelt durch einen Bügel, der die Spitzen der beiden Stifte verbindet. Ein Rändelknopf an dem nach außen durchgeführten Teil eines Stiftes gestattet die gekoppelte Drehung beider Stifte um die gemeinsame Längsachse. Die Löcher, in denen die Stifte durch die Wand der Wanne geführt sind, sind durch Präzisionsarbeit und Ölung wasserdicht.

Die Modellfaser wird mit ihren Enden auf den beiden gegenüberliegenden Metallspitzen so befestigt, daß sie die gemeinsame Achse der beiden Stifte fortsetzt über die Lücke zwischen den beiden Spitzen hinweg. Die Modellfaser dreht sich also um ihre Längsachse, wenn die Stifte gedreht werden. Die kontrahierte, mit Formaldehyd fixierte Faser wird zunächst in Glycerin übertragen und dann auf die beiden Spitzen mit Cellonlack aufgeklebt.

Die Untersuchung der Auslöschrichtungen fand in verdünnter Pufferlösung (s. unter Lösungen) statt.

Die Kontraktion der Fasern: Damit sich die Fasern bei der Kontraktion nicht krümmen, werden sie mit den Enden auf zwei Celluloidplättchen befestigt, deren Abstand verändert werden kann. Bei der Kontraktion wird dieser Abstand so verringert, daß der Faden bei der Verkürzung gestreckt bleibt. Die Enden der Modellfaser werden mit Cellonlack befestigt.

M essungen: Die Faserbreite und -dicke wird nach H. H. W e be ${ }^{3}{ }^{3}$ gemessen. Die Vergrößerung war 740 -fach.

Die Messungen der Doppelbrechung geschahen mit Berek-Kompensator bei 740-facher Vergrößerung in Grünlicht von $\sim 533 \mathrm{~m} \mu$. Lichtfilter ${ }^{9}$ : In $100 \mathrm{ccm} \mathrm{H}_{2} \mathrm{O} 60 \mathrm{~g}$ $\mathrm{CuCl}_{2} \cdot 2 \mathrm{H}_{2} \mathrm{O}$; in $100 \mathrm{ccm} \mathrm{H}_{2} \mathrm{O} 10 \mathrm{~g} \mathrm{Kaliummonochromat.}$ Schichtdicke jeweils $20 \mathrm{~mm}$. Niedervoltlampe.

Die Brechungsindices der Imbibitionsmittel wurden für die angegebene Wellenlänge laufend mit einem AbbéRefraktometer kontrolliert.

Der Eiweißgehalt wurde als Trockenvolumen mikroskopisch bestimmt. Das Volumen der luftrockenen Faser wurde mit dem Volumen im Bad verglichen. Der Wassergehalt der lufttrockenen Faser in Abhängigkeit vom Wasserdampfgehalt der Luft wurde den Eichkurven von H. H. W e b e r ${ }^{3}$ und $\mathrm{N}$ o $\mathrm{ll}-\mathrm{W}$ e b e $\mathrm{r}^{6}$ entnommen. Der Wasserdampfgehalt der Luft selbst wurde gleichzeitig mit der mikroskopischen Bestimmung des Trockenvoli1mens mit einem Taupunktshygrometer (Aspirationspsychometer) bestimmt. Der Anteil des Eiweißes am Volumen der Modellfaser in verdünnter Pufferlösung beträgt im Mittel 15\%.

9 E. Abderhalden, Handbuch der biolog. Arbeitsmethoden 1928, Abt. II, S. 1218.
Berechnungen: Da die Fasern in den verschiedenen Imbibitionsmitteln in wechselndem Umfange quellen, wird die Doppelbrechung immer auf den Normalwert von 15\% Eiweiß umgerechnet. Hierfür gilt:

1. Die Eigendoppelbrechung ist ceteris paribus der Eiweißkonzentration direkt proportional.

2. Die Formdoppelbrechung ist in erster Annäherung ceteris paribus proportional dem Produkt $v_{1} \cdot v_{2}$. Hier bedeutet $v_{1}$ das Volumen der Eiweiß-Stäbchen, $v_{2}$ das Volumen der Zwischenräume. Wird nämlich in der W i e n e r schen Formel für Stäbchenmischkörper

$$
n_{\mathrm{a}}-n_{0}=\frac{v_{1} v_{2}\left(n_{1}{ }^{2}-n_{2}{ }^{2}\right)^{2}}{\left(n_{\mathrm{a}}+n_{0}\right)\left[\left(1+v_{1}\right) n_{2}{ }^{2}+v_{2} n_{1}{ }^{2}\right]}
$$

in erster Annäherung $n_{\mathrm{a}}+n_{\mathrm{o}}$ durch $2 n_{\mathrm{m}}$ und $n_{1}{ }^{2}$ und $n_{2}{ }^{2}$ im Nenner durch $n_{\mathrm{m}}{ }^{2}$ ersetzt, so nimmt sie die Form an

$$
n_{\mathrm{a}}-n_{0}=v_{1} v_{2} \frac{\left(n_{1}{ }^{2}-n_{2}^{2}\right)^{2}}{4 n_{\mathrm{m}}{ }^{3}} .
$$

In dieser Formel ist $n_{\mathrm{m}}=v_{1} \cdot n_{1}+v_{2} \cdot n_{2}$.

Der Fehler der Annäherung gegenüber der korrekten Wienerschen Formel beträgt für das Imbibitionsmittel Alkohol zwischen 1\% und 2\%, für Monobromnaphthalin weniger als $1 \%$, wenn das Doppelbrechungsminimum bei 1,56 liegt.

Chemikalien und Lösungẹn

Die Glycerinmischungen wurden aus wäßrigem Phosphatpuffer und Glycerinum bidestillatum p. a. 1,23 Merck gemischt. Unter Berücksichtigung des Wassergehaltes des Merckschen Glycerins betrug der Glyceringehalt an Volumenprozent

1. für die Extraktionsmischung $30 \%$,

2. für die Mischung, in der die Präparate aufbewahrt wurden und mit der sie zum Aufkleben durchtränkt wurden, $87 \% *$,

3. für die Mischung, in der die dünnen Faserbündel in Einzelfasern zerlegt wurden, $18 \%$.

Die Pufferkonzentrationen wurden so gewählt, daß die Phosphatkonzentration in allen Glycerinmischungen und in wäßriger Lösung $1 \cdot 10^{-2}-m$. und das $p_{\mathrm{H}} \sim 7$ betrug. In den verdünnten Glycerinlösungen und in der wäßrigen Lösung verhielt sich $\mathrm{Na}_{2} \mathrm{HPO}_{4}: \mathrm{NaH}_{2} \mathrm{PO}_{4}$ wie $1: 1$, in der konzentrierten Glycerinmischung wie $1: 1,6$. Die wäßrige Pufferlösung enthielt außerdem $m / 1000 \mathrm{MgCl}_{2}$.

Die Adenosintriphosphat-Konzentration war bei der Kontraktion der Fasern für die Doppelbrechungsanalyse $\sim 5 \cdot 10-3-m$., für die Stauchungsuntersuchungen $\sim 8 \cdot 10^{-3}-m$.; Formaldehyd und Imbibitionsmittel waren M ercksche Präparate p. a., nur der Äthylalkohol

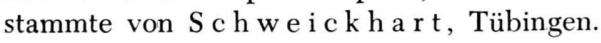

Herrn Professor H. H. W e be r bin ich für die Anregung zur Arbeit und die Hilfe bei ihrer Durchführung zu großem Dank verpflichtet.

* Das gleiche gilt für die Publikation von A. W eb e r 5 , in der die Konzentration versehentlich mit $96 \%$ angegeben ist. 